\title{
Exigências térmicas de Aedes (Stegomyia) albopictus Skuse, 1894 (Diptera, Culicidae) em condições de laboratório ${ }^{1}$
}

\author{
Daniéla C. Calado ${ }^{2}$ \\ Mário Antonio Navarro-Silva ${ }^{3}$
}

\begin{abstract}
Thermal requirements of Aedes (Stegomyia) albopictus Skuse, 1894 (Diptera, Culicidae) under laboratory conditions. Allochtonous species of Ae. albopictus in the American continent can be found in fourteen Brazilian states about fifteen years had been elapsed since the first report of the presence of this species in Brazilian territory. Considering its potential epidemiological importance and its adaptation to several habitats, it was determined, for this species, the threshold temperature and the thermal constant for egg, larval and pupal stages under laboratory conditions under four constant temperatures and 12:12 hours light-dark photoperiod. The threshold temperature for the egg phase and for the first instar were quite similar: $9.07{ }^{\circ} \mathrm{C}(\mathrm{K}=214.46$ degree days $)$ and $9.23{ }^{\circ} \mathrm{C}(\mathrm{K}=36.64$ degree days $)$, respectively. For $2^{\text {nd }}$, $3^{\text {rd }}$ and $4^{\text {th }}$ instar, the basal-temperature was higher, oscilating between $12.26{ }^{\circ} \mathrm{C}$ and $13.95{ }^{\circ} \mathrm{C}$. The threshold temperature for the complete larval stage and for the pupal stage were $12.03{ }^{\circ} \mathrm{C}(\mathrm{K}=99.48$ degree days $)$ and $11.87{ }^{\circ} \mathrm{C}(\mathrm{K}=32.40$ degree days $)$ for males and $11.95{ }^{\circ} \mathrm{C}(\mathrm{K}=110.11$ degree days $)$ and $11.60{ }^{\circ} \mathrm{C}(\mathrm{K}=35.30$ degree days $)$ for females, respectively.
\end{abstract}

KEYwords. Aedes albopictus; biology; Diptera; thermal requirements.

\section{INTRODUÇÃO}

Aedes albopictus é espécie alóctone para o continente americano e decorridos aproximadamente quinze anos do primeiro registro desta espécie no Brasil, sua presença foi assinalada em quatorze estados brasileiros (Gomes et al. 1999). A importância epidemiológica desta espécie está relacionada à transmissão do vírus da dengue (IBÁÑEZ-BERnAL et al. 1997), da febre amarela e de outros agentes etiológicos como a Dirofilaria immitis (Leidy, 1856) (KoNISHI 1989).

Em território brasileiro, aspectos do desenvolvimento de Ae. albopictus foram abordados por XAVIER et al. (1991) a partir de exemplares capturados no Campus Ecológico da Universidade Federal de Minas Gerais, e porGomes et al. (1995) que avaliaram, sob condições de campo, o desenvolvimento de imaturos em criadouros naturais e artificiais. Em outras regiões geográficas, considerável número de informações tem sido gerado em relação à biologia de Ae. albopictus, e diferenças têm sido detectadas entre as populações (Tsuda et al. 1994).

Considerando a grande importância epidemiológica de $A e$. albopictus e sua facilidade de dispersão e adaptação aos mais variados habitats, o conhecimento das exigências térmicas dos estágios imaturos poderá auxiliar no melhor entendimento de sua biologia e no levantamento do maior número de informações que possam ser utilizadas em programas e estratégias de controle.

Tendo em vista que, dentre os componentes de um modelo de previsão da ocorrência de insetos, a temperatura ocupa lugar de destaque, por trata-se de um dos fatores climáticos que mais diretamente afeta estes organismos (HADDAD \& PARRA 1984), o presente trabalho teve como objetivo determinar a temperatura base e a constante térmica para os estágio de ovo, larva e pupa de Ae. albopictus, sob condições de laboratório, em quatro temperaturas constantes e fotofase de 12 horas.

\section{MATERIALE MÉTODOS}

A população de Ae. albopictus utilizada no desenvolvimento dos experimentos foi obtida a partir de colônia estabelecida com imaturos, coletados em recipiente artificial na área urbana da cidade de Registro, São Paulo, Brasil. A colônia é mantida, desde março de 1999 , em sala climatizada de $25 \pm 3^{\circ} \mathrm{C}$, umidade relativa do ar de $85 \pm 10 \%$ e fotoperíodo natural. Amostra de exemplares de Ae. albopictus da colônia encontram-

1. Contribuição no 1336 do Departamento de Zoologia, Universidade Federal do Paraná.

2. Curso de Pós-graduação em Entomologia, Departamento de Zoologia, Universidade Federal do Paraná. Bolsista do CNPq. Endereço eletrônico: dcalado@bio.ufpr.br

3. Departamento de Zoologia, Universidade Federal do Paraná. Caixa Postal 19020, 81.531-980, Curitiba-PR, Brasil. Endereço eletrônico: mnavarro@bio.ufpr.br 
Tabela I. Temperatura base ( $\mathrm{Tb})$, Constante térmica $(\mathrm{K})$, equação da reta e coeficiente de determinação $\left(\mathrm{R}^{2}\right)$, para as fases imaturas de Aedes albopictus, sob quatro temperaturas constantes, fotofase de 12 horas e umidade relativa de $70-85 \%$.

\begin{tabular}{lcccc}
\hline Fases do ciclo biológico & $\mathrm{Tb}\left({ }^{\circ} \mathrm{C}\right)$ & $\mathrm{K}(\mathrm{GD})^{*}$ & Equação da reta & $\mathrm{R}^{2}$ \\
\hline Ovo & 9,07 & 214,46 & $1 / \mathrm{D}=-0,042274+0,004662 * \mathrm{~T}$ & 0,995 \\
Primeiro instar & 9,23 & 36,64 & $1 / \mathrm{D}=-0,252022+0,027293 * \mathrm{~T}$ & 0,925 \\
Segundo instar & 13,95 & 11,21 & $1 / \mathrm{D}=-1,243131+0,089173 * \mathrm{~T}$ & 0,954 \\
Terceiro instar & 12,95 & 16,53 & $1 / \mathrm{D}=-0,783281+0,060510^{* \mathrm{~T}}$ & 0,983 \\
Quarto instar & 12,26 & 41,49 & $1 / \mathrm{D}=-0,295613+0,024100^{* \mathrm{~T}}$ & 0,994 \\
Larva (fềmeas) & 11,95 & 110,11 & $1 / \mathrm{D}=-0,108554+0,009082 * \mathrm{~T}$ & 0,992 \\
Larva (machos) & 12,03 & 99,48 & $1 / \mathrm{D}=-0,120942+0,010051^{*} \mathrm{~T}$ & 0,991 \\
Pupa (fêmeas) & 11,60 & 35,30 & $1 / \mathrm{D}=-0,328724+0,028331^{*} \mathrm{~T}$ & 0,974 \\
Pupa (machos) & 11,87 & 32,40 & $1 / \mathrm{D}=-0,366306+0,030868 * \mathrm{~T}$ & 0,973 \\
\hline
\end{tabular}

$* \mathrm{GD}=$ graus-dias

se depositados na Coleção de Entomologia Pe. Jesus Santiago Moure, Departamento de Zoologia da Universidade Federal do Paraná, Curitiba (DZUP).

A determinação da temperatura base (Tb) e o valor da constante térmica $(\mathrm{K})$, expressa em graus-dias (GD) foram obtidos de acordo com HADDAD \& PARRA (1984), a partir do "Método da Hipérbole". Segundo estes autores, a utilização deste método só é satisfatória quando os dados experimentais são obtidos, no mínimo, em quatro temperaturas diferentes e constantes. A equação: $1 / D=a+b T$ foi obtida através da regressão linear entre a temperatura e a velocidade de desenvolvimento (1/dias), onde: $1 / D$ é a velocidade de desenvolvimento; $a$, o coeficiente linear da reta; $b$, o coeficiente angular da reta e $T$, a temperatura. Para a obtenção da temperatura base, assumiu-se que: $1 / D=0$. Logo, $T b=-a / b{ }^{\circ} \mathrm{C}$ e $K=1 / b$ $G D$ (GD = graus-dias). O coeficiente de determinação (R2) foi obtido para a verificação da proporção da variação de 1/D que é explicada pela regressão.

Em consequência da exigência do método de análise, foram utilizadas quatro temperaturas constantes $\left(15^{\circ} \mathrm{C}, 20^{\circ} \mathrm{C}, 25^{\circ} \mathrm{Ce}\right.$ $30^{\circ} \mathrm{C}$ ) estabelecidas em câmaras climatizadas modelo 347-CDG marca FANEM, fotofase de 12 horas e umidade relativa entre $70-85 \%$. As observações, da temperatura e da umidade relativa do ar, foram realizadas durante a fotofase.

O período de incubação e a viabilidade dos ovos foram avaliados a partir de 200 ovos, divididos em quatro lotes de 50, mantidos em recipientes de polietileno com capacidade para $120 \mathrm{ml}$. Em cada recipiente foram colocados $90 \mathrm{ml}$ de água de torneira submetida à aeração e adicionado alimento constituído de $0.05 \mathrm{~g}$ de ração para peixes TetraMin. Diariamente, foi acrescentada água para evitar a adesão dos ovos às paredes dos recipientes. A troca do meio líquido foi realizada a cada dois dias. Os frascos foram monitorados uma vez ao dia, para retirada de larvas e contagem das larvas eclodidas.

Para determinar a duração do período larval, foram utilizadas 200 larvas de primeiro instar de mesma idade, divididas em quatro lotes de 50 imaturos. As larvas foram mantidas em bandejas plásticas translúcidas e retangulares $(21,4 \times 14,1 \times 5,7 \mathrm{~cm})$, com capacidade para $900 \mathrm{ml}$. Em cada bandeja foram colocados $400 \mathrm{ml}$ de água de torneira submetida à aeração e alimento. A troca da água e o fornecimento de alimento foram realizados a cada dois dias.

Duas vezes ao dia (7:30 e 18:30 h), as exúvias larvais foram retiradas e contabilizadas, anotando-se o dia e período (manhã ou tarde) no qual ocorreu a ecdise. Foi estipulado que o intervalo manhã-tarde, corresponderia a 0,5 dia e o intervalo manhãmanhã ou tarde-tarde, a 1 dia. Este procedimento foi adotado devido à curta duração do segundo e terceiro instares, sob as temperaturas de $25^{\circ} \mathrm{C}$ e $30^{\circ} \mathrm{C}$. O cálculo do período médio de duração de cada instar foi realizado a partir da subtração das datas entre duas ecdises. A duração do período larval para machos e fêmeas foi obtida a partir da subtração da data do final do quarto instar da data de eclosão das larvas. Após a passagem para o estágio de pupa, os imaturos foram

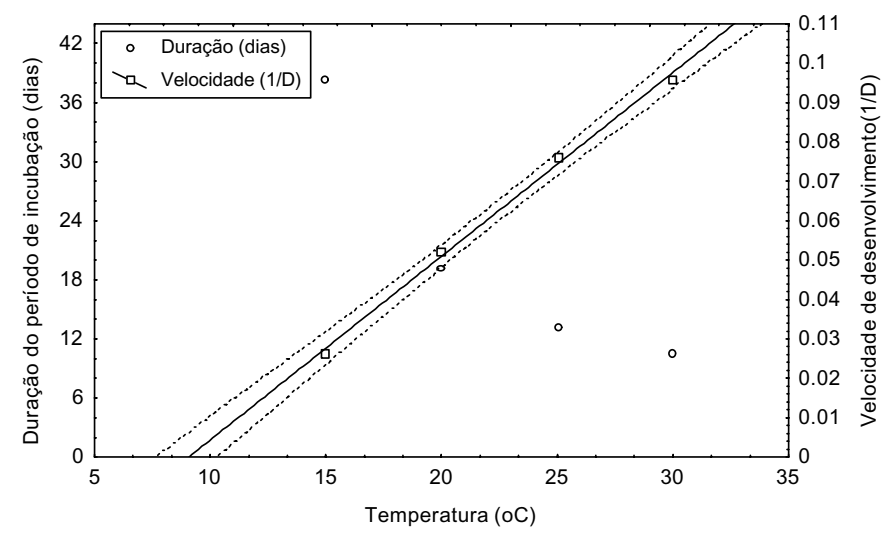

Fig.1. Duração do período de incubação (dias) e velocidade de desenvolvimento (1/D) para o estágio de ovo de Aedes albopictus, sob quatro temperaturas constantes, fotoperíodo de 12 horas e umidade relativa de $70-85 \%$. 

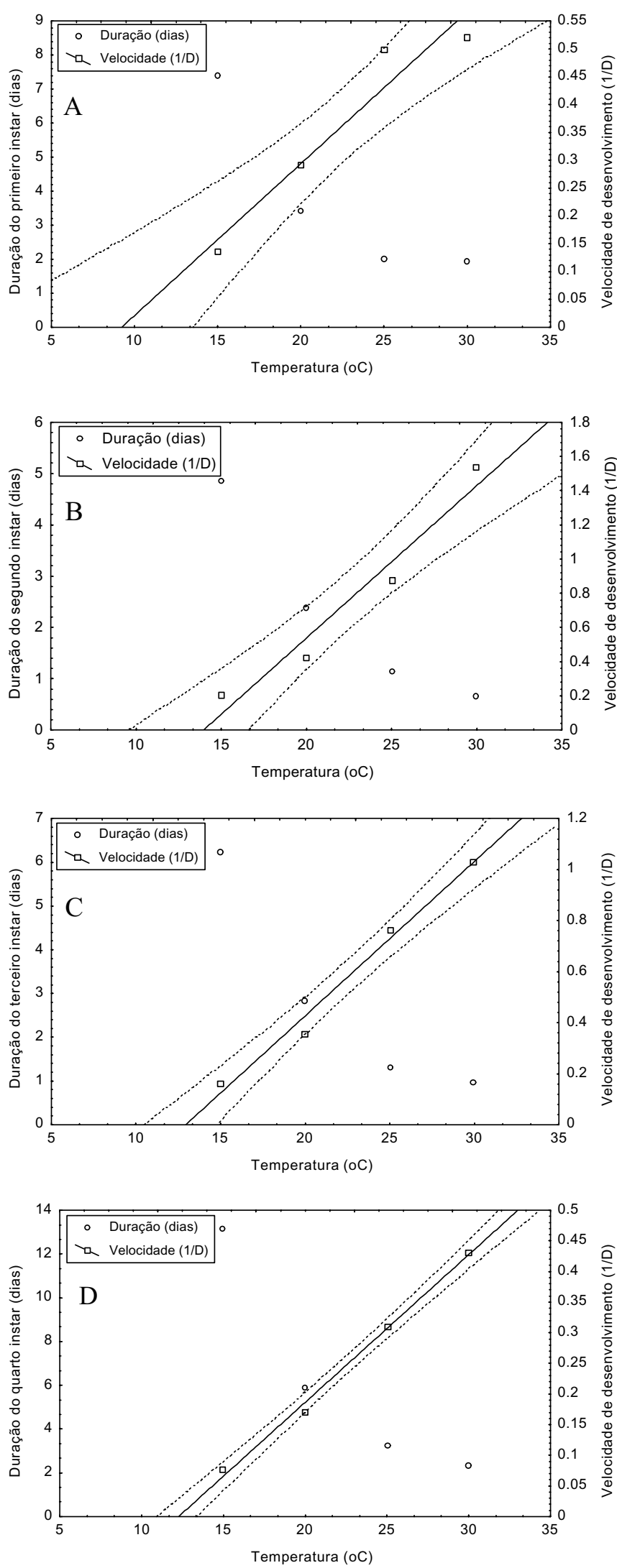

Fig. 2. Duração em dias e velocidade de desenvolvimento (1/D) para o $1^{\circ}$ (A) , 2ㅇ (B), 3o (C) e 4 (D) instar de Aedes albopictus, sob quatro temperaturas constantes, fotofase de 12 horas e umidade relativa de 70 $85 \%$. individualizados, possibilitando a verificação do sexo de cada indivíduo.

A separação de pupas em recipientes individuais foi utilizada para permitir o cálculo desse período para machos $\mathrm{e}$ fêmeas. Foram utilizadas pupas recém formadas mantidas em aproximadamente $40 \mathrm{ml}$ de água de torneira submetida à aeração. Os recipientes foram cobertos com filme plástico de PVC para evitar a fuga dos indivíduos emergidos. Duas vezes ao dia, as exúvias pupais foram retiradas e realizada a separação por sexo.

\section{RESULTADOS E DISCUSSÃO}

As análises de regressão linear entre as velocidades médias de desenvolvimento de cada estágio e as temperaturas constantes utilizadas, mostraram-se altamente significativas, indicando que esta variável apresenta grande influência sobre o ciclo aquático de Ae. albopictus e que a velocidade de desenvolvimento é inversamente proporcional à temperatura (Tabela 1).

Os valores de temperatura base para o estágio de ovo e para o primeiro instar foram muito semelhantes, indicando que estas fases constituem as mais resistentes às baixas temperaturas. Para os demais instares $\left(2^{\circ}, 3^{\circ} \mathrm{e} 4^{\circ}\right)$ a temperatura base foi superior, oscilando entre $12,26^{\circ} \mathrm{C}$ e $13,95^{\circ} \mathrm{C}$. As temperaturas-base para os estágios larval e pupal, foram de $12,03^{\circ} \mathrm{C}$ e $11,87^{\circ} \mathrm{C}$ para machos e de $11,95^{\circ} \mathrm{C}$ e $11,60^{\circ} \mathrm{C}$ para fêmeas, respectivamente (Tabela I e Figs. 1-3). Os resultados obtidos para o estágio larval são próximos à constante térmica (K) de 105,26 graus-dias acima da temperatura base de $10,37^{\circ} \mathrm{C}$ obtida por LeE (1994), mas diferem da temperatura base de 8,81 ${ }^{\circ} \mathrm{C}$ obtida por Teng \& Apperson (2000). Estas pequenas variações quanto às características biológicas podem ser resultantes das adaptações ecológicas exibidas pela espécie (TsudA et al. 1994), tendo em vista sua rápida dispersão para vários continentes e a exploração de uma grande diversidade de recursos.

Em relação aos diferentes valores de K e de temperaturasbase obtidos para os estágios de desenvolvimento, estes podem ser resultado das diferenças estruturais e fisiológicas próprias de cada estágio, que garantem à espécie a capacidade de adaptação às variações ambientais. Assim, em regiões de inverno rigoroso, a população possivelmente permanece na fase de ovo até que a elevação da temperatura atinja o limiar de desenvolvimento.

Em Madagascar, a distribuição desta espécie está limitada às áreas onde a temperatura média mensal é superior a $12{ }^{\circ} \mathrm{C}$ (FonTENILLE \& RHODAIN 1989). Quanto à distribuição temporal, Tома et al. (1982) observaram que em regiões temperadas do Japão, onde a temperatura durante o inverno frequentemente atinge $0{ }^{\circ} \mathrm{C}, A e$. albopictus é encontrada somente na fase de ovo, enquanto em áreas de clima subtropical, onde a temperatura durante o inverno é de aproximadamente de $17^{\circ} \mathrm{C}$, esta espécie também pode ser encontrada no estágio larval. Este último caso é válido para as regiões mais frias do território brasileiro. Na região sul do Brasil, embora as temperaturas 

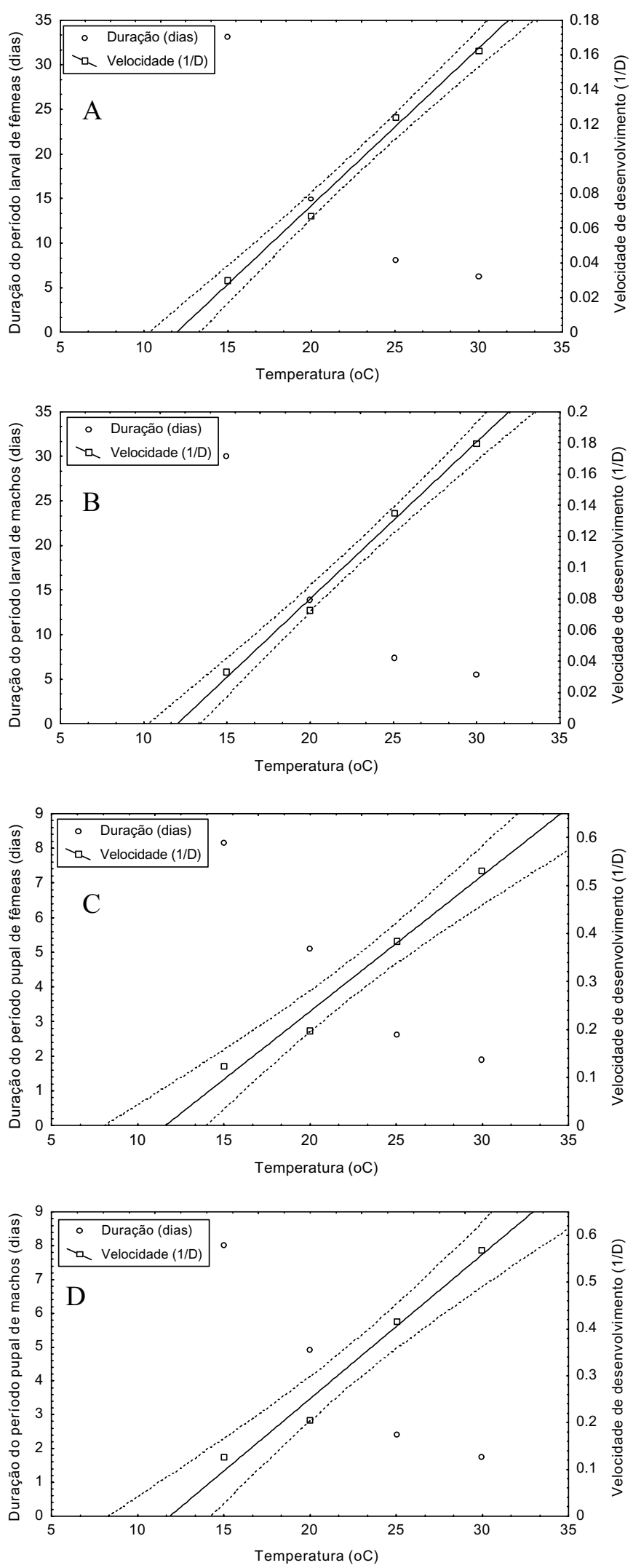

Fig. 3. Duração em dias e velocidade de desenvolvimento (1/D) para o estágio larval de fêmeas (A) e machos (B), e para o estágio pupal de fêmeas (C) e machos (D) de Aedes albopictus, sob quatro temperaturas constantes, fotofase de 12 horas e umidade relativa de $70-85 \%$. médias anuais sejam superiores às temperaturas-base obtidas para os estágios de desenvolvimento de Ae. albopictus, os meses que compreendem o inverno apresentam dias sucessivos de temperaturas abaixo do limiar, o que pode implicar na desaceleração da velocidade de desenvolvimento ou até mesmo na morte de larvas e pupas. A ocorrência frequente de temperaturas abaixo deste limiar, pode constituir fator limitante ao crescimento e à distribuição espacial e temporal das populações de Ae. albopictus.

Cabe ressaltar que, além da resistência de cada estágio frente às baixas temperaturas, outros fatores contribuem para a sobrevivência ou para o desenvolvimento contínuo de uma espécie durante os meses mais frios. HANSON \& CRAIG JR (1995) apontam que, em condições naturais, ovos de Ae. albopictus não sobrevivem em microhabitats relativamente expostos, durante o período do inverno em Indiana (EUA). Entretanto, em criadouros mais protegidos, seja pela vegetação, solo ou outros materiais, ou mesmo no ambiente intradomiciliar, a variação da temperatura do microhabitat pode ser menor, ampliando as chances de sobrevivência durante o período do inverno. Mesmo criadouros artificiais presentes em áreas de vegetação mais densa, dependendo de sua constituição podem apresentar maior resistência à queda da temperatura da água em relação à do ar ( SiLVA \& Lozovei 1996).

A manutenção de criadouros artificiais adequados aos estágios imaturos, durante períodos de temperaturas acima do limiar de desenvolvimento, pode propiciar a manutenção da população em nível elevado, tornando-se mais um elemento a ser considerado na definição de estratégias de controle da espécie. Pode-se inferir que o nível populacional de $A e$. albopictus estará mais baixo quando a temperatura estiver próxima a $9^{\circ} \mathrm{C}$ para a população estabelecida na região do Vale do Ribeira, local de origem da população analisada.

Agradecimentos. À Fundação Nacional de Saúde e Organização Panamericana de Saúde, pelo apoio financeiro. A Mestranda Andreia A. Barbosa (Curso de Pós-Graduação em Entomologia, UFPR), pela coleta do material utilizado na implementação das colônias.

\section{REFERÊNCIAS}

Fontenille, D. \& F. Rhodain. 1989. Biology an distribuition of Aedes albopictus and Aedes aegypti in Madagascar. Journal American Mosquito Control Association 5(2): 219-225.

Gomes A. De C.; S. L. D. Gotlieb; C. De A. M. Marques; M. B. de Paula \& G. R. A. M. MARques. 1995. Duration of larval and pupal development stages of Aedes albopictus in natural and artificial containers. Revista de Saúde Pública 29(1):15-19.

Gomes, A. De C.; M. D. Bitencourt; D. Natal; P. L. S. Pinto; L. F. Mucci; M. B. de Paula; P. R. Urbinatti \& J. M. S. Barata. 1999. Aedes albopictus em área rural do Brasil e implicações na transmissão de febre amarela silvestre. Revista de Saúde Pública 33(1):95-97.

HadDad, D. M. L. \& J. R. P. PARRA. 1984. Métodos para estimar os limites térmicos e a faixa ótima de desenvolvimento das diferentes fases do ciclo evolutivo dos insetos. Piracicaba, FEALQ, 12p.

Hanson, S. M. \& G. B. Craig Jr. 1995. Aedes albopictus (Diptera: Culicidae) eggs: field survivorship during northern Indiana winters. Journal of 
Medical Entomology 32(5): 599-604.

ibáñez-Bernal, S; B. Briseno; J. P. Mutebi; E. Argot; G. Rodriguez; C. Martinez-Campos; R. Paz; P. de La F. Roman; R. Tapia-Conyer \& A. FLISSER. 1997. First record in America of Aedes albopictus naturally infected with dengue virus during the 1995 outbreak at Reynosa, Mexico. Medical Veterinary Entomology 11: 305-309.

KonISHI, E. 1989. Culex tritaeniorhynchus and Aedes albopictus (Diptera: Culicidae) as natural vector of Dirofilaria immitis (Spirurida: Filariidae) in Miki City, Japan. Journal of Medical Entomology 26(4):295300 .

LeE, S. J. 1994. Development of eggs, larvae and pupae of Aedes albopictus (Skuse) (Diptera: Culicidae). Zhonghua Kunchong 14: 13-32.

Silva, M. A. N. DA \& A. L. Lozover. 1996. Criadouros de imaturos de mosquitos (Diptera, Culicidae) introduzidos em mata preservada na área urbana de Curitiba, Paraná, Brasil. Revista Brasileira de
Zoologia 13(4): 1023-1042.

Teng, H. \& C. S. Apperson. 2000. Developmemt and survival of immature Aedes albopictus and Aedes triseriatus (Diptera: Culicidae) in the laboratory: Effects of density, food and competition on response to temperature. Journal of Medical Entomology 37(1): $40-52$.

Toma, T; S. SAкамото \& I. Myagi. 1982. The seasonal appearance of Aedes albopictus in Okinawajima, the Ryukiu Arquipelago, Japan. Mosquito News 42(2): 179-183.

Tsuda, Y.; M. TAKagi; A. SuzuKi \& Y. WADA. 1994. A comparative study on life table characteristics of two strains of Aedes albopictus from Japan and Thailand. Tropical Medicine 36(1): 15-20.

Xavier, G. V.; D. P. Neves \& R. F. DA Silva. 1991. Ciclo Biológico do Aedes albopictus (Diptera-Culicidae), em laboratório. Revista Brasileira de Biologia 51(3): 647-650. 